Heinen, Simon; Frenz, Martin; Schlick, Christopher M.

\title{
Bildung für nachhaltige Entwicklung in der Gebäudeenergieberatung - Entwicklung eines Kompetenzmodells für die Förderung reflexiver
}

\section{Handlungsfähigkeit}

Seifried, Jürgen [Hrsg.]; Faßhauer, Uwe [Hrsg.]; Seeber, Susan [Hrsg.]: Jahrbuch der berufs- und wirtschaftspädagogischen Forschung 2014. Opladen [u.a.] : Verlag Barbara Budrich 2014, S. 67-79. (Schriftenreihe der Sektion Berufs- und Wirtschaftspädagogik der Deutschen Gesellschaft für Erziehungswissenschaft (DGfE); 217)

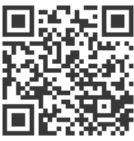

Quellenangabe/ Reference:

Heinen, Simon; Frenz, Martin; Schlick, Christopher M.: Bildung für nachhaltige Entwicklung in der Gebäudeenergieberatung - Entwicklung eines Kompetenzmodells für die Förderung reflexiver Handlungsfähigkeit - In: Seifried, Jürgen [Hrsg.]; Faßhauer, Uwe [Hrsg.]; Seeber, Susan [Hrsg.]: Jahrbuch der berufs- und wirtschaftspädagogischen Forschung 2014. Opladen [u.a.] : Verlag Barbara Budrich 2014, S. 67-79 - URN: urn:nbn:de:0111-pedocs-97268 - DOI: 10.25656/01:9726

https://nbn-resolving.org/urn:nbn:de:0111-pedocs-97268

https://doi.org/10.25656/01:9726

in Kooperation mit / in cooperation with:

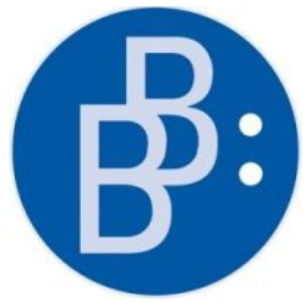

https://www.budrich.de

\section{Nutzungsbedingungen}

Dieses Dokument steht unter folgender Creative Commons-Lizenz: http://creativecommons.org/licenses/by-nc-nd/3.0/de/deed - Sie dürfen das Werk bzw. den Inhalt unter folgenden Bedingungen vervielfältigen, verbreiten und öffentlich zugänglich machen: Sie müssen den Namen des Autors/Rechteinhabers in der von ihm festgelegten Weise nennen. Dieses Werk bzw. dieser Inhalt darf nicht für kommerzielle Zwecke verwendet werden und es darf nicht bearbeitet, abgewandelt oder in anderer Weise verändert werden.

Mit der Verwendung dieses Dokuments erkennen Sie die Nutzungsbedingungen an.

\section{Terms of use}

This document is published under following Creative Commons-License: http://creativecommons.org/licenses/by-nc-nd/3.0/de/deed.en - You may copy, distribute and transmit, adapt or exhibit the work in the public as long as you attribute the work in the manner specified by the author or licensor. You are not allowed to make commercial use of the work or its contents. You are not allowed to alter, transform, or change this work in any other way.

By using this particular document, you accept the above-stated conditions of use.

\section{Kontakt / Contact:}

peDOCS

DIPF | Leibniz-Institut für Bildungsforschung und Bildungsinformation Informationszentrum (IZ) Bildung

E-Mail: pedocs@dipf.de

Internet: www.pedocs.de

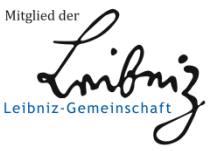




\section{Jahrbuch der berufs- und wirtschaftspädagogischen Forschung 2014}

Jürgen Seifried, Uwe Faßhauer Susan Seeber (Hrsg.)

DGE Deutsche Gesellschaft 
Schriftenreihe der Sektion

Berufs- und Wirtschaftspädagogik der Deutschen Gesellschaft für Erziehungswissenschaft (DGfE) 
Jürgen Seifried

Uwe Faßhauer

Susan Seeber (Hrsg.)

\section{Jahrbuch der berufs- und wirtschaftspädagogischen Forschung 2014}

Verlag Barbara Budrich

Opladen • Berlin • Toronto 2014 
Bibliografische Information der Deutschen Nationalbibliothek

Die Deutsche Nationalbibliothek verzeichnet diese Publikation in der Deutschen Nationalbibliografie; detaillierte bibliografische Daten sind im Internet über http://dnb.d-nb.de abrufbar.

(C) Dieses Werk ist im Verlag Barbara Budrich erschienen und steht unter folgender Creative Commons Lizenz: http://creativecommons.org/licenses/by-nc-nd/3.0/de/ Verbreitung, Speicherung und Vervielfältigung erlaubt, kommerzielle Nutzung und Veränderung nur mit Genehmigung des Verlags Barbara Budrich.

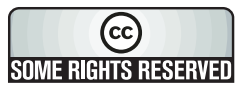

Dieses Buch steht im OpenAccess Bereich der Verlagsseite zum kostenlosen Download bereit (http://dx.doi.org/10.3224/84740164)

Eine kostenpflichtige Druckversion (Printing on Demand) kann über den Verlag bezogen werden. Die Seitenzahlen in der Druck- und Onlineversion sind identisch.

$$
\begin{array}{ll}
\text { ISBN } & 978-3-8474-0164-3 \text { (Paperback) } \\
\text { eISBN } & \mathbf{9 7 8 - 3 - 8 4 7 4 - 0 4 4 1 - 5 ~ ( e B o o k ) ~} \\
\text { DOI } & 10.3224 / 84740164
\end{array}
$$

Umschlaggestaltung: bettina lehfeldt graphic design, Kleinmachnow

Typografisches Lektorat: Judith Henning, Hamburg

Verlag Barbara Budrich, http://www.budrich-verlag.de 


\section{Inhaltsverzeichnis}

Vorwort 9

\section{Teil I: Perspektiven der historischen Berufsbildungsforschung}

Frank-Lothar Kroll

Möglichkeiten und Notwendigkeiten historiographischen Arbeitens in der Berufs- und Wirtschaftspädagogik........................................................ 1

Volker Bank, Annekathrin Lehmann

Theodor Franke. Sächsischer Pionier wirtschaftspädagogischen

Denkens in Deutschland

Marcel Schweder

Lehrerarbeit im Strafvollzug - Ein Diskurs aus historischer Sicht

\section{Teil II: Kompetenzmodellierung, -messung und -förderung}

Eveline Wittmann, Ulrike Weyland, Annette Nauerth, Ottmar Döring, Simone Rechenbach, Julia Simon, Iberé Worofka Kompetenzerfassung in der Pflege älterer Menschen - Theoretische und domänenspezifische Anforderungen der Aufgabenmodellierung 53

Simon Heinen, Martin Frenz, Christopher M. Schlick

Bildung für nachhaltige Entwicklung in der Gebäudeenergieberatung - Entwicklung eines Kompetenzmodells für die Förderung reflexiver Handlungsfähigkeit

Diana Stuckatz, Cornelia Wagner

Qualifizierungsangebote in der Pflegehilfe für Personen mit geringen Grundbildungskenntnissen - Empirische Studien zur Entwicklung von Lehr-Lern-Umgebungen und Arbeitsmaterialien. 81 


\section{Teil III: Gestaltung und Analyse von Lehr-Lern-Prozessen}

Eva Höpfer, Andrea Reichmuth, Doreen Holtsch, Franz Eberle

Wer sieht was? - Zum Umgang mit unterschiedlichen Sichtweisen auf

Unterricht am Beispiel des kaufmännischen Berufsschulunterrichts 95

Mandy Hommel

Sozial geteilte Reflexion - eine explorative Studie im

Mathematikunterricht. 109

Gerhard Minnameier, Rico Hermkes

„Kognitive Aktivierung“ und „konstruktive Unterstützung“ als Lehr-

Lern-Prozess-Größen - Eine Konzeption im rechnungswesen-

didaktischen Kontext

\section{Teil IV: Lehrerbildung und pädagogische Professionalität}

Nicole Kimmelmann, Johannes Lang

Lehramtsstudierende mit Migrationshintergrund und ihre

Schwierigkeiten an der Universität

Robert W. Jahn

Stützlehrer als neuer pädagogischer Profi in der Beruflichen Bildung?! .... 147

Sabrina Berg

Pädagogische Praxis und Reproduktion sozialer Ungleichheit - zur

Berücksichtigung sozialer Herkunft im Wirtschaftsunterricht..... 161

\section{Teil V: Perspektiven der Berufsbildungsforschung}

\section{Miriam Voigt}

Neo-institutionalistische und mikropolitische Prozesse in

Schulentwicklungsprojekten

Lara Forsblom, Lucio Negrini, Jean-Luc Gurtner \& Stephan Schumann

Lehrvertragsauflösungen und die Rolle der betrieblichen Auswahl von

Auszubildenden 
Marius R. Busemeyer

Organisierte Interessen, Parteipolitik und institutioneller Wandel im deutschen Berufsbildungssystem

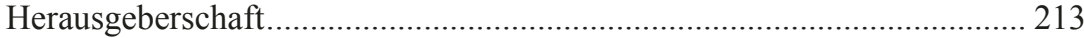

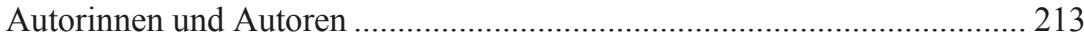




\title{
Bildung für nachhaltige Entwicklung in der Gebäudeenergieberatung - Entwicklung eines Kompetenzmodells für die Förderung reflexiver Handlungsfähigkeit
}

\author{
Simon Heinen, Martin Frenz, Christopher M. Schlick
}

\section{Einleitung und Ausgangslage}

Ziel des Beitrages ist es, ein didaktisches Leitbild der Nachhaltigkeit in der beruflichen Weiterbildung auf Aufgabenebene zu verankern. Dies geschieht beispielhaft für die Erwerbstätigkeit Gebäudeenergieberatung, die v. a. von Handwerksmeistern aus den Gewerken Bau und Ausbau, Sanitär-HeizungKlima, Elektro oder dem Schornsteinfegerhandwerk mit entsprechender Fortoder Weiterbildung ausgeübt wird.

Für die Gebäudeenergieberatung fehlt derzeit ein anforderungsgerechtes, situationsorientiertes Konzept nachhaltiger Bildung. Existierende Bildungsangebote fokussieren den Erwerb theoriebasierten Fachwissens in formellen Lernprozessen. Die Kompetenzförderung vor dem Hintergrund eines bildungstheoretischen Leitgedankens, der z.B. das Lösen von Zielkonflikten oder das Gestalten von Interaktionsprozessen in Beratungssituationen umfasst, wird hingegen vernachlässigt (Heinen et al. 2013).

In diesem Beitrag wird als Grundlage für die Förderung der Handlungsfähigkeit von Gebäudeenergieberatern zunächst vor dem Hintergrund einer Bildung für nachhaltige Entwicklung (Kapitel 2) die Erarbeitung von Handlungsfeldern der Gebäudeenergieberatung vorgestellt (Kapitel 3). Auf Grundlage dieser empirischen Studien wird ein Kompetenzmodell entwickelt (Kapitel 4) und anhand eines Beispiels ein konkreter Vorschlag für die didaktische Gestaltung von Weiterbildungsmodulen anhand des Kompetenzmodells gegeben (Kapitel 5).

\section{Bildung für nachhaltige Entwicklung}

Übergeordnetes Ziel einer Bildung für nachhaltige Entwicklung ist es, den Menschen zu befähigen, aktiv und konstruktiv an der gesellschaftlichen Entwicklung mitzuwirken und diese zu gestalten (Kandler/Tippelt 2011). Für die 
Kompetenzentwicklung wird in diesem Zusammenhang das Bildungsverständnis nach De Haan (2002) zugrunde gelegt. Demnach ist Bildung geprägt durch drei Dimensionen: Offenheit gegenüber neuen Erfahrungen und Wissen, Zukunftsfähigkeit (Erfahrungen und wahrgenommene Möglichkeiten nutzen für einen positiven Umgang mit Unsicherheit zukünftiger Wandlungsprozesse) und Reflexion (Wandlungsprozesse bezogen auf sich selbst und die Umwelt wahrnehmen, reflektieren und das eigene Handeln modifizieren). Im Sinne des Nachhaltigkeitsgedankens ist hierbei der Erwerb bzw. die Förderung von Kompetenzen zur Wahrnehmung, Lösung und insbesondere Reflexion von Problemen erforderlich (De Haan/Harenberg 1999).

Für die Weiterbildung in der Gebäudeenergieberatung muss dieses allgemeine Ziel der Förderung reflexiver Handlungsfähigkeit konkretisiert werden. Oberstes Ziel der beruflichen Bildung ist die Ermöglichung und Förderung beruflicher Handlungsfähigkeit für die auszuführenden Tätigkeiten (vgl. §1 BBiG). Für Energieberatende bedeutet diese Konkretisierung, sich permanent mit den Aspekten „Ökonomische Leistungsfähigkeit“, „Ökologische Verträglichkeit" und "Soziale/Gesellschaftliche Verantwortung“" auseinanderzusetzen, daraus entstehende Anforderungen im Denken und Handeln $\mathrm{zu}$ berücksichtigen und auftretende Widersprüchlichkeiten beim Entwickeln von Lösungen abzuwägen.

Um für die Weiterbildung die zu fördernden Kompetenzen beschreiben zu können, müssen zunächst die spezifischen Anforderungen in der Erwerbstätigkeit vor diesem Hintergrund empirisch erfasst und in Handlungsfeldern beschrieben werden.

\section{Studien der Qualifikationsforschung und Entwicklung von Handlungsfeldern}

Um die zu fördernden Kompetenzen für die Gestaltung von Bildungsangeboten systematisch beschreiben zu können, muss zunächst mit einer handlungsorientierten Beschreibung der Tätigkeiten eine entsprechende Grundlage geschaffen werden (vgl. Reetz/Seyd 2006, Siebert 2012). Hierzu bieten tätigkeitsbezogene Methoden der Qualifikationsforschung einen guten $\mathrm{Zu}-$ gang zur Erschließung und zur Analyse der Tätigkeiten (Buchmann 2006). Entsprechend wurden für die Gebäudeenergieberatung Studien der Qualifikationsforschung durchgeführt. Die erhobenen Tätigkeiten wurden anschließend in Handlungsfeldern systematisiert und in Expertenworkshops validiert (Heinen et al. 2011).

Zentrales Ergebnis ist, dass die Arbeitsaufgaben in der Gebäudeenergieberatung häufig durch offene Problemstellungen, hohe Komplexität, dialo- 
gisch-interaktive Arbeitshandlungen und das Auftreten von Unplanbarkeiten geprägt sind (Tabelle 1).

\section{Tab. 1: Studien der Qualifikationsforschung und zentrale Erkenntnisse}

\begin{tabular}{|c|c|c|}
\hline Untersuchung & Ergebnis & Zentrale Erkenntnis \\
\hline $\begin{array}{l}\text { Dokumenten- } \\
\text { analyse }\end{array}$ & $\begin{array}{l}\text { Tätigkeitsanfor- } \\
\text { derungen, derzei- } \\
\text { tige Bildungs- } \\
\text { strukturen und } \\
\text { Bildungsinhalte }\end{array}$ & $\begin{array}{l}\text { - Energieberater benötigen umfassendes fachliches Wissen und } \\
\text { Können in Breite und Tiefe aus verschiedenen Gewerken und } \\
\text { müssen dieses miteinander vernetzen. } \\
\text { - Es besteht eine hohe Dynamik in der Domäne durch ständig } \\
\text { neue Richtlinien, gesetzliche Vorschriften etc. } \\
\text { - Bisher sind Bildungsstrukturen und -inhalte auf die Vermitt- } \\
\text { lung fachwissenschaftlicher Grundlagen ausgerichtet. }\end{array}$ \\
\hline $\begin{array}{l}\text { Arbeitsprozess- } \\
\text { studien }\end{array}$ & $\begin{array}{l}\text { Beschreibung } \\
\text { Typischer } \\
\text { Arbeitsabläufe }\end{array}$ & $\begin{array}{l}\text { - Energieberater müssen in hohem Maße konzeptionell denken } \\
\text { und problemlösend handeln. } \\
\text { - Viele Arbeitsaufgaben sind zieloffen und von Widersprüchen } \\
\text { geprägt. } \\
\text { - Es muss in hohem Maße Interaktionsarbeit mit Kunden, Ge- } \\
\text { schäftspartnern etc. geleistet werden. }\end{array}$ \\
\hline $\begin{array}{l}\text { Fleishman- Job } \\
\text { Analyse System } \\
\text { (F-JAS) }\end{array}$ & $\begin{array}{l}\text { Eigenschaftsbe- } \\
\text { zogene } \\
\text { Anforderungen }\end{array}$ & $\begin{array}{l}\text { - Energieberater benötigen ein breites Spektrum kognitiver, so- } \\
\text { zialer und interpersoneller Fähigkeiten und Fertigkeiten. }\end{array}$ \\
\hline
\end{tabular}

Quelle: Heinen 2012, Heinen et al. 2013

Bei der Bewältigung dieser Arbeitsaufgaben treten häufig Zielkonflikte im Spannungsfeld zwischen ökologischer Verträglichkeit, ökonomischer Leistungsfähigkeit und sozialer/gesellschaftlicher Verantwortung auf, die ein Energieberater abwägen und für sich bewerten muss, um Entscheidungen zu fällen und entsprechend handeln zu können. Bei diesen Problemlöseprozessen findet „Interaktionsarbeit" nach Böhle (2006) und Hacker (2009) statt. Für eine erfolgreiche Energieberatung gilt es nicht nur, eine fundierte Lösung für ein Problem zu erarbeiten, sondern den Kunden hinsichtlich verschiedener möglicher Lösungen zu beraten, widersprüchliche Anforderungen aufzuzeigen und die bevorzugte Lösung dem Kunden auch als hochwertigen Vorschlag zu vermitteln.

Abbildung 1 zeigt die entwickelten Handlungsfelder der Gebäudeenergieberatung. Zentrale Tätigkeiten von Gebäudeenergieberatern sind das Erfassen der energetischen Qualität von Gebäuden (bspw. Gebäudehülle, technische Gebäudeausstattung etc.), das Beraten von Kunden im Hinblick auf technisch, ökologisch, gesellschaftlich und ökonomisch sinnvolle Modernisierungsmaßnahmen, das Beraten der Kunden bei der Beantragung von staatlichen Fördermitteln zur Modernisierung von Wohngebäuden etc. Ebenso werden auch Leistungen wie das Erstellen von und Beratung zu Energiekonzepten einschließlich Begleitung von Maßnahmen des Energie- und 
Facility-Management während der Nutzung des Gebäudes angeboten (s. Frenz/Marfels 2011).

\section{Abb. 1: Handlungsfelder der Gebäudeenergieberatung}

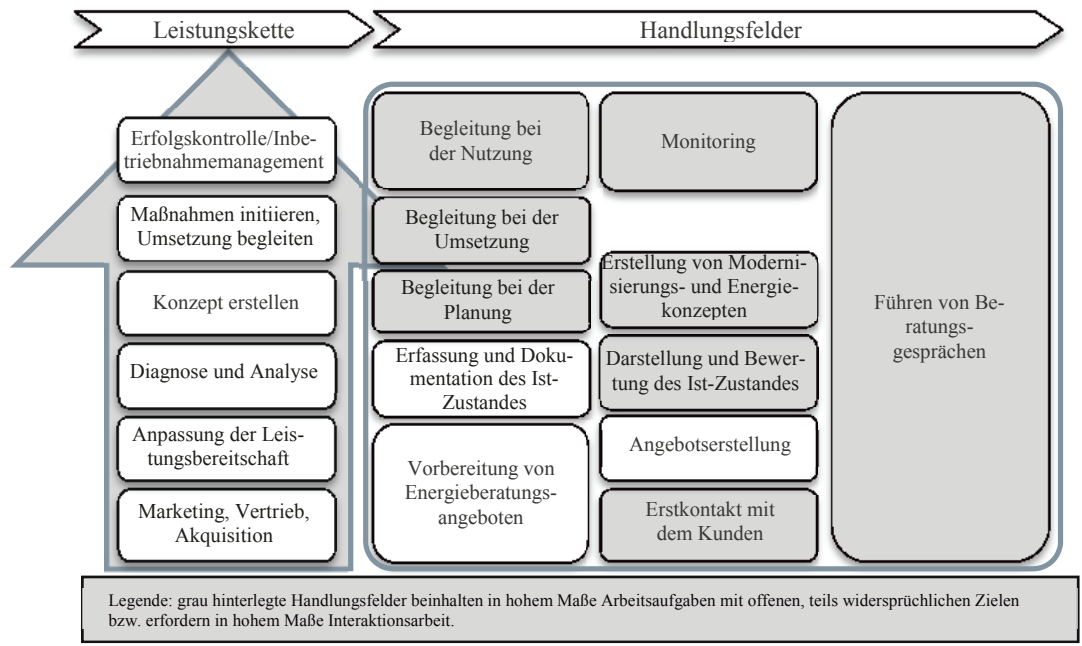

Quelle: Heinen et al. 2011

\section{Modell der Handlungskompetenz von Gebäudeenergieberatern}

Aufbauend auf der Erfassung und handlungsorientierten Beschreibung der spezifischen Anforderungen in der Erwerbstätigkeit kann nun die zu fördernde Handlungskompetenz von Gebäudeenergieberatern vor dem Hintergrund einer Bildung für nachhaltige Entwicklung beschrieben werden. Diese Beschreibung im Sinne didaktischer Ziele erfolgt im entwickelten Modell hinsichtlich der Dimensionen Handlungsbezug, Kompetenzart sowie Niveau.

Handlungsbezug: Entsprechend der Tätigkeitsanforderungen der Handlungsfelder (Kap. 2) muss ein Energieberatender konkrete Handlungssituationen einer Bildung für nachhaltige Entwicklung gestalten. Die Handlungsfähigkeit eines Energieberaters schließt insbesondere ein reflexives $\mathrm{Ab}$ wägungsverhalten im Sinne der Nachhaltigkeit in der konkreten Handlungssituation ein.

Kompetenzart: Die Differenzierung der Handlungsfähigkeit erfolgt unter Bezug auf die anthropologisch-pädagogische Position von Heinrich Roth 
(1976; vgl. Reetz 1999) im Sinne eines Subjekt-Objekt-Bezuges nach den Kompetenzarten Fach-, Sozial- und Selbstkompetenz.

Das vorgestellte Konzept ist somit uneingeschränkt anschlussfähig an das Kompetenzverständnis des Deutschen Qualifikationsrahmens, welches durch einen engen Bezug zum Konzept der beruflichen Handlungsfähigkeit im dualen System letztlich auch eine anthropologisch-pädagogische Position aufweist. Diese Anschlussfähigkeit ist für eine spätere, mögliche Einstufung der Erwerbstätigkeiten in der Gebäudeenergieberatung in den DQR und somit einer Charakterisierung der Anforderungsstrukturen aufgrund der angestrebten Lernergebnisse wichtig. Der gewählte Zugang bietet aber gegenüber dem Kompetenzkonzept des DQR zwei entscheidende Vorteile. Zum einen erlaubt es das Konzept nach Roth, sich stärker von der dominanten Position der Lernergebnisorientierung zu lösen und somit stärker den Prozess der Kompetenzentwicklung für beraterisches Handeln und Problemlösen zu fokussieren. Zum anderen ist es aus analytischer Sicht notwendig, gleichberechtigt zwischen Fach-, Selbst- und Sozialkompetenz zu trennen. Die eindeutige Differenzierung von Fach-, Selbst- und Sozialkompetenz erscheint hinsichtlich der Differenzierung von Niveaus nach dem im folgenden $\mathrm{Ab}-$ schnitt vorgestellten Reflexionsmodell von Tiefel (2004) analytisch notwendig.

Niveau: Die Beschreibung der Handlungsfelder verdeutlicht, dass in fast allen Handlungsfeldern ein Höchstmaß an Reflexion erforderlich ist. Unter diesem Fokus werden für das Kompetenzmodell Niveaus der Reflexion unterschieden (Abb. 2). Grundlage für deren Definition sind die vier Reflexionsmodi für beraterisches Handeln nach Tiefel (2004). Der Reflexionsmodus beschreibt, wie offen ein Individuum ein bestimmtes Problem reflektiert, d. h. durch Reduzierung der Problemkomplexität (Stabilisierung) oder Sich-Einlassen auf mögliche Problemszenarien (Innovation) (s. Abb. 2, links). Es wird differenziert zwischen jeweils zwei stabilisierenden und zwei innovierenden Modi, die jeweils durch den Reflexionsfokus und das Reflexionswissen beschreibbar sind (s. Abb. 2, Mitte). Der Reflexionsfokus beschreibt die Integration unterschiedlicher Wahrnehmungsperspektiven in die Gestaltung einer Lösung, d.h. ob sich die Problembewältigung eher an bewährte Lösungsschemata hält (stabilisierend), oder innovative, offene Lösungswege notwendig sind. Reflexionswissen beschreibt die Art und Funktion des Wissenszugangs, also ob Wissen eher reproduziert wird oder für den Gegenstandsbereich transformiert werden muss, sowie den Umgang mit Nichtwissen. Stabilisierende Modi sind geprägt durch einen starken Bezug zu standardisierten Handlungsschemata. Die innovativen Modi erfordern eine höhere Kreativität und den Umgang mit Ungewissheiten. Für diese vier Stufen wurden vier allgemeine Problemtypen definiert, welche die Zieloffenheit und den Interaktionsaufwand berücksichtigen (s. Abb. 2, rechts). 
Gestaltung des Neuen - Offene Probleme (zieloffen, Zielkonflikte, unklarer Interaktionsausgang): Dieser Problemtyp erfordert eine kreative Nutzung von oder eine Entwicklung neuer Vorgehensweisen zur Schaffung neuer Lösungen oder eine kreative Gestaltung einer dynamischen Interaktion mit mehreren Projektbeteiligten mit unterschiedlichen Interessen unter permanent wechselnden Interessen.

Variation des Alten - Geschlossene Probleme ohne klare Regeln: Dieser Problemtyp erfordert bewusste Adaption von Vorgehensweisen auf neue Sachverhalte oder eine dynamische Interaktion unter permanent wechselnden, aber wahrnehmbaren Interessenslagen mit einem oder mehreren Projektbeteiligten.

Bewusste Anpassung - Algorithmisches Vorgehen mit Regeln: Es werden Regeln genutzt, bekannte Lösungen adaptiert oder die Interaktion mit verschiedenen Projektpartnern und klaren Interessen gestaltet.

Routinierte Anpassung - Eindeutige Regeln: Es werden für bekannte Lösungswege Rezepte angewendet oder mit einem Projektpartner und klaren Interessen interagiert.

Abb. 2: Niveaus der Reflexion und Problemtypen beraterischen Handelns

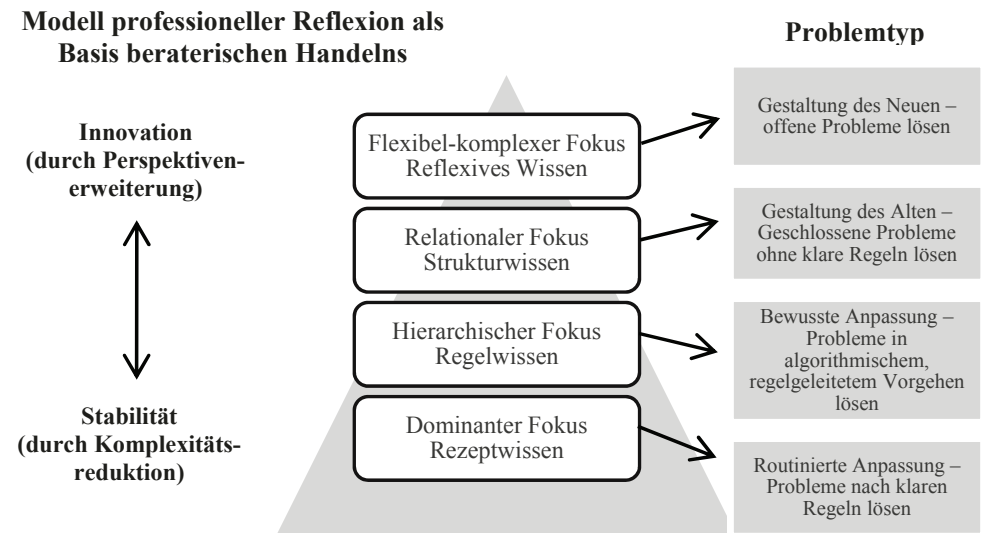

Quelle: in Anlehnung an Tiefel 2004

Abbildung 3 zeigt das Kompetenzmodell, welches nachfolgend erläutert wird. Für ein Weiterbildungsmodul lässt sich durch eine Beschreibung der drei Dimensionen Handlungssituation, Kompetenzart und Niveau ein konkretes Problem beschreiben, anhand dessen ein Lernprozess ausgestaltet wird. Dadurch können die didaktischen Ziele begründet werden und anschließend die methodische Ausgestaltung des Weiterbildungsmoduls erfolgen. 
Die Bildung für nachhaltige Entwicklung dient zum einen als Strukturierungsschema für die Arbeitsaufgaben in der jeweiligen Handlungssituation. Die Tätigkeitsanforderungen werden dadurch unter dem Fokus der Dimensionen Ökologie, Ökonomie und Gesellschaft dargelegt. Dadurch wird der Handlungsbezug für die Beschreibung der didaktischen Ziele und Inhalte durch die Ausgestaltung eines Problemtypen eines Weiterbildungsmoduls sichergestellt. Zum anderen bietet sie als bildungstheoretischer Leitgedanke ein grundsätzliches Reflexionsschema für die Kompetenzentwicklung. Dies ermöglicht eine systematische Beschreibung und Konkretisierung der zu fördernden Kompetenzen für den gewählten Problemtyp.

Abb. 3: Kompetenzmodell

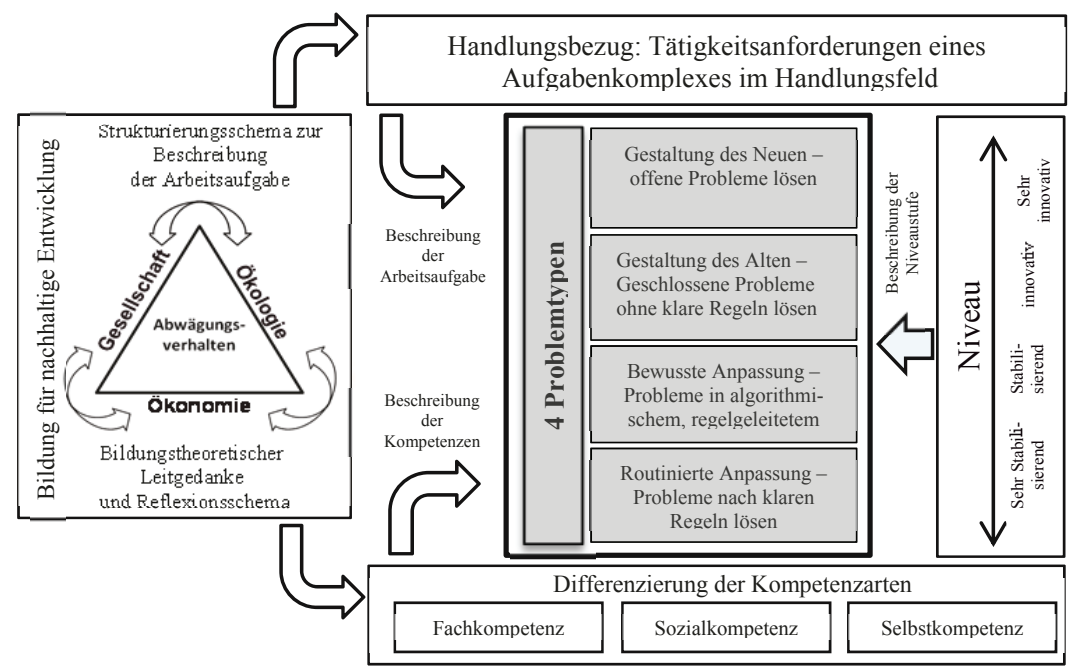

Quelle: eigene Darstellung

Um die Effektivität des Konzeptes bzgl. des Ziels der Förderung reflexiver Handlungsfähigkeit zu bewerten, muss eine Erfassung der erworbenen Handlungsfähigkeit sowie eine Evaluation der durchgeführten Weiterbildung erfolgen (vgl. Reetz 2011, Heidegger 2006). Mit Anschlussfähigkeit an das zuvor dargestellte Kompetenzmodell wurde hierzu ein Konzept zur Diagnose von Energieberatungskompetenzen entwickelt (vgl. Djaloeis et al. 2011). Dieses Konzept greift die über das Kompetenzmodell operationalisierten Handlungssituationen auf und ermöglicht anhand einer entwickelten Referenzlösung der Arbeitsaufgabe eine strukturierte Beschreibung der Problemlösefähigkeit von Energieberaterinnen und Energieberatern hinsichtlich einer sach- und fachgerechten Lösung sowie einer Bearbeitung der Handlungssituation im Sinne des Nachhaltigkeitsgedankens. Über eine Selbst- so- 
wie Fremdevaluation durch Experten und Teilnehmende erfolgt anschließend eine Reflexion und qualitiative Bewertung, in welchem Ausmaß die angestrebte Entwicklung der reflexiven Handlungsfähigkeit erreicht wurde.

\section{Didaktische Gestaltung von Weiterbildungsmodulen zur Förderung reflexiver Handlungsfähigkeit}

Im Folgenden wird exemplarisch anhand eines Weiterbildungsmoduls gezeigt, wie auf Grundlage des entwickelten Kompetenzmodells die Konzeption entsprechender Module theoretisch gestützt werden kann (vgl. Heinen/ Frenz 2013). Die Beschreibung der didaktischen Ziele für das Weiterbildungsmodul „Beratung einer Stadtverwaltung zum öffentlichen Bad"erfolgt anhand der Problemtypen des Kompetenzmodells. Die anschließende Gestaltung des Weiterbildungsmoduls folgt gängigen Ansätzen aus der Weiterbildung: Handlungsorientiertes, situiertes Lernen unter Einbezug konstruktivistischer Ansätze, der beruflichen Wirklichkeit und der biografischen Erfahrungen der Teilnehmer (Siebert 2012). Abschließend werden die prototypische Erprobung des Moduls vorgestellt und Evaluationsergebnisse diskutiert.

Für das Handlungsfeld „Führen von Beratungsgesprächen“ sind Beratungen von Kunden, z. B. von privaten Hausbesitzern oder in diesem Falle einer Kommune, zu erstellten Energiekonzepten eine zentrale Aufgabe. Ein erstelltes Energiekonzept berücksichtigend, steht hier die Interaktion mit anderen Personen, also Kunden, Vermietern, Geschäftspartnern etc., und somit die Förderung von Sozialkompetenz im Vordergrund. Je nach Beratungsaufgabe kann die bei der Beratung zu berücksichtigende Gruppe größer oder kleiner sein. Die Problemtypen werden zunächst im Wesentlichen aufgrund der unterschiedlichen Interaktionserfordernisse des zu wählenden Niveaus differenziert. Das nachfolgende Weiterbildungsmodul basiert auf einer realen Handlungssituation eines Projektteams, das dieses komplexe Problem des Typs Gestaltung des Neuen zu bewältigen hatte.

Ausgangslage: Die Energiekosten einer Kleinstadt, einem anerkannten Erholungsort, wirkten sich sehr negativ auf die Haushaltslage aus. Im Rahmen der Fördermaßnahme „Kommunaler Klimaschutz“ des Bundesumweltministeriums (BMU) wurden durch einen TGA-Planer Einsparmöglichkeiten und ein Energiekonzept für die örtlichen Therme, einem Spaß- und Freizeitbad, entwickelt. Nun soll die Stadtverwaltung durch einen Energieberater für das vorliegende Konzept hinsichtlich der Qualität und Nachhaltigkeit des Energiekonzeptes beraten werden. Bei diesem Projekt gibt es verschiedene Projektbeteiligte, deren unterschiedliche Interessen es zu berücksichtigen gilt (Abb. 4). 
Bei der Stadtverwaltung besteht offensichtlich die Haltung, dass Einzelaktionen, eine Ingenieur-Planung und Einzelinvestitionen ausreichend seien, und eine fachliche Erfolgskontrolle nicht erforderlich. Eine aktive Mitwirkung wird als nicht notwendig erachtet. Die Interessen der Fördermittelgeber (Land und BMU) liegen eher darin, im Sinne der Fördermaßnahme eine nachhaltige Lösung umzusetzen. Die technische Planung durch ein eingebundenes Ingenieurbüro fokussiert wiederum die Planung und den Verkauf neuer technischer Anlagen.

\section{Abb. 4: Vorgehensweise im Weiterbildungsmodul und beraterische Interaktionen}

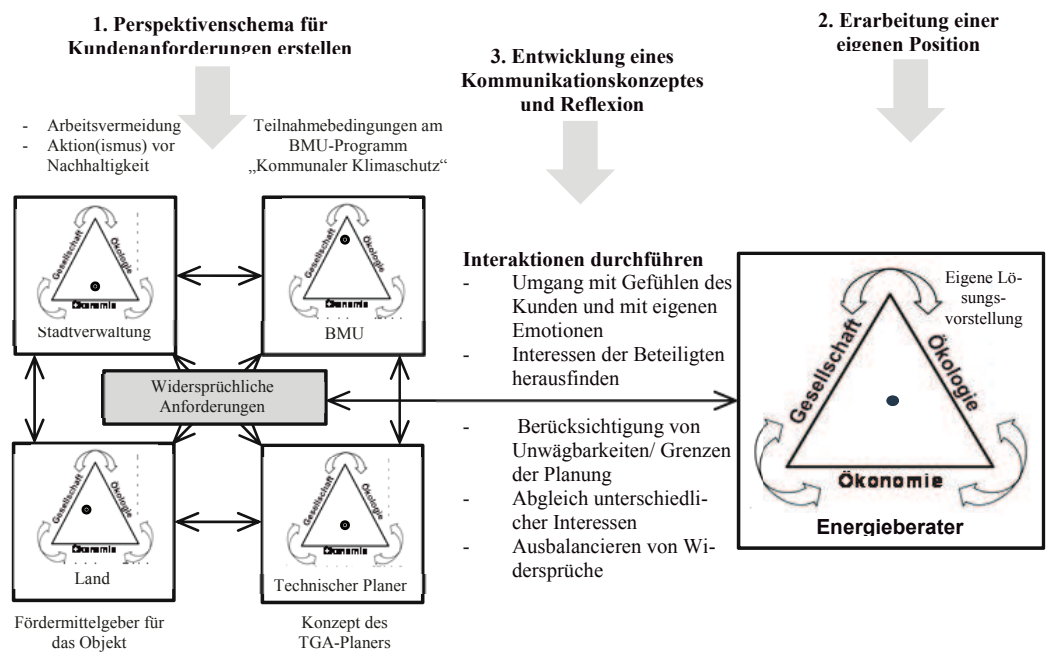

Quelle: eigene Darstellung

In dieser Handlungssituation soll die Stadtverwaltung eines anerkannten Kurortes hinsichtlich der Qualität und Nachhaltigkeit eines vorliegenden Energiekonzeptes für das städtische Spaß- und Freizeitbad beraten werden.

Didaktische Ziele: Die Weiterbildungsteilnehmer sind in der Lage, aufgrund der Ausgangslage die Anforderungen und Interessen der beteiligten Partner zu erschließen, anhand eines erstellten Energiekonzeptes fach- und sachgerecht $\mathrm{zu}$ reflektieren und entsprechende Handlungsstrategien für die Beratung der Stadtverwaltung zu entwickeln. Die besondere Herausforderung besteht darin, Interessen und Absichten aller Beteiligten (Fördermittelgeber, TGA-Planer, Stadtverwaltung etc.) systematisch unter ökologischen, ökonomischen und gesellschaftlichen Aspekten aufzunehmen und Interessens- und Zielkonflikte zwischen den Beteiligten aufdecken zu können. Sie sind in der 
Lage, einen Lösungsvorschlag, der von allen Beteiligten getragen wird, und eine entsprechende Kommunikationsstrategie zu entwickeln.

Aus dieser Handlungssituation könnten auch für die anderen Niveaus Probleme abgeleitet und Module gestaltet werden. Beispiele hierfür wären: Routinierte Anpassung: Hier könnte das zu bewältigende Problem beispielsweise die Beratung eines Mitarbeiters der Stadtverwaltung über die Auswertung eines Blower-Door-Tests, um Leckagen in der Gebäudehülle aufzuspüren, sein. Es gibt hier klare Regeln, so erfolgt für eine als undicht indentifizierte Türe zwingend die Empfehlung, diese abzudichten.

Bewusste Anpassung: Hierfür könnte das Problem die Beratung der Stadtverwaltung über einen erstellten Energiebedarfsausweis für Nichtwohngebäude darstellen. Der Stadtverwaltung wird die energetische Bewertung des Gebäudes erläutert und Beratungsempfehlungen ausgesprochen. Hierfür gibt es entsprechend der geltenden Energieeinsparverordnung (EnEV) Vorgaben zur Ausstellung, zur Verwendung und zu den Modernisierungsempfehlungen.

Variation des Alten: Dieser Problemtyp könnte anhand einer Beratung der Stadtverwaltung und der Objektverantwortlichen im Rahmen des zu erstellenden Energiekonzeptes für das örtliche Spaß- und Freizeitbad ausgestaltet werden. Die Entwicklung der Modernisierungsvorschläge muss mit den beteiligten Partnern unter Berücksichtigung der spezifischen, sich verändernden Interessen aller Beteiligten abgestimmt werden. Die Modernisierungsvorschläge müssen zwar individuell ausgestaltet und ausgehandelt werden, dabei kann jedoch auf Erfahrungen, z.B. bekannte Vorgehensweisen und Methoden aus anderen Beratungen zurückgegriffen werden und diese für den vorliegenden Fall adaptiert werden.

Durchführung, Bewertung und Evaluation: Die Handlungssituation wurde innerhalb einer dreitägigen internen Weiterbildung eines Energieberatungsdienstleisters in einem halbtägigen Modul erprobt. Die Weiterbildungsteilnehmer erarbeiteten zunächst in Zweiergruppen anhand der vorliegenden Fallbeschreibung, vorgestellt durch einen der damaligen Projektbearbeiter, ein Perspektivenschema mit den Interessen und Absichten aller Beteiligten und beschrieben diese unter ökologischen, ökonomischen und gesellschaftlichen Aspekten. Sie legten Interessens- und Zielkonflikte zwischen den Beteiligten dar. In Abhängigkeit vom jeweiligen Projektbeteiligten reflektierten die Teilnehmer, in welcher Rolle sie sich jeweils sehen und welche Möglichkeiten der Kommunikation sie sehen. Die identifizierten Konfikte und aufgestellten Bewältigungsstrategien wurden dem Plenum vorgestellt und anhand der Erfahrungen eines Projektbearbeiters reflektiert. Abschließend erfolgte eine weitere Diskussion und Reflektion anhand eigener Erfahrungen der Teilnehmenden. Unter Nutzung von Grundlagen zur Kommunikation und zu Rollenmodellen erarbeiteten Sie ihre Rolle als Energieberater. 
Im Anschluss an die Weiterbildung erfolgten separate, qualitative Evaluationsgespräche mit den Teilnehmenden und den damaligen Projektbearbeitern als Betreuer der gesamten Weiterbildungsmaßnahme. Das Modul ist gut geeignet zur Förderung der angestrebten reflexiven Handlungsfähigkeit und soll für diese interne Weiterbildung weiter eingesetzt werden. Mit Grundlagen zur Kommunikation (z. B. Nutzung des Vier-OhrenModells), zu Rollenmodellen und mit der Entwicklung eines Perspektivenschemas wurden den Teilnehmenden wichtige Methoden und Instrumente für die Projektbearbeitung und die individuelle Strukturierung komplexer Beratungssituationen vermittelt. Auch die Möglichkeit zur Reflexion eigener Beispiele wurde als sehr positiv bewertet.

Die prototypische Erprobung des Konzeptes kann also als erfolgreich bezeichnet werden. Um die Effektivität dieses Weiterbildungmoduls für das Ziel der Förderung reflexiver Handlungsfähigkeit empirisch stärker zu fundieren, müssten im Anschluss die weiteren Weiterbildungen evaluiert werden, um die Erkenntnisse weiter generalisieren zu können. Zudem müsste der Einsatz des zum Zeitpunkt der Weiterbildung noch in der Erprobung befindlichen Diagnosekonzeptes optimiert werden. Ebenso steht eine Transferevaluation der Anwendbarkeit in der Praxis noch aus.

\section{Zusammenfassung}

Vor dem Hintergrund der in der Einleitung aufgezeigten Defizite in der Weiterbildung von Gebäudeenergieberatern wurde in diesem Beitrag unter Bezug auf einschlägige Studien der Qualifikationsforschung ein Kompetenzmodell zur Förderung reflexiver Handlungskompetenz entwickelt. Dieses Kompetenzmodell nutzt den theoretischen didaktsichen Ansatz einer Bildung für nachhaltige Entwicklung zur Beschreibung didaktischer Ziele für ein situiertes Konzept der Kompetenzförderung. Anhand eines Beispiels wurde gezeigt, wie mit dem Kompetenzmodell die Planung einer Weiterbildungsmaßnahme theoretisch gestützt werden kann. Dieses Beispiel hebt sich deutlich von zur Zeit vorherrschenden Weiterbildungsangeboten in der Gebäudeenergieberatung $\mathrm{ab}$, da es durch den bildungstheoretischen Leitgedanken einer Bildung für nachhaltige Entwicklung begründet ist und sich auf erhobene Arbeitsprozesse der Energieberatung und nicht auf unverbundene Wissensgebiete - z.B. der Bauphysik und der Anlagen- und Gerätetechnik - bezieht. 


\section{Literatur}

Böhle, F. (2006): Typologie und strukturelle Probleme von Interaktionsarbeit. In: Böhle, F./Glaser, J. (Hrsg.): Arbeit in der Interaktion - Interaktion als Arbeit. Wiesbaden: VS, S. 325-347.

Buchmann, U. (2006): Empirische Qualifikationsforschung und ihr Beitrag zur Curriculumkonstruktion - Eine Kommentierung deutschsprachiger Literatur. In: Pätzold, G./Rauner, F. (Hrsg.): Qualifikationsforschung und Curriculumentwicklung. ZBW Beiheft 19. Stuttgart: Steiner, S. 235-255.

Djaloeis, R./Frenz, M./Heinen, S./Schlick, C. (2011): Diagnose von Energieberatungskompetenz. In: Faßhauer, U./Fürstenau, B./Wuttke, E. (Hrsg.): Grundlagenforschung zum dualen System und Kompetenzentwicklung in der Lehrerbildung. Opladen: Budrich, S.131-142.

De Haan, G. (2002): Die Kernthemen der Bildung für nachhaltige Entwicklung In: Zeitschrift für Entwicklungspädagogik, H. 1, S. 13-20.

De Haan, G./Harenberg, D. (1999): Bildung für nachhaltige Entwicklung. Gutachten zum Programm. In: BLK-Materialien zur Bildungsplanung und zur Forschungsförderung, 72. Bd.. Bonn

Frenz, M./Marfels, K. (2011): Professionalisierung der Dienstleistung in der Energieberatung - Strategien auf individueller und organisatorischer Ebene. In: Gatermann, I./Fleck, M. (Hrsg.): Mit Dienstleistungen die Zukunft gestalten. Frankfurt und New York: Campus, S. 131-139.

Hacker, W. (2009): Arbeitsgegenstand Mensch:Psychologie dialogisch-interaktiver Erwerbsarbeit. Lengerich u.a: Pabst.

Heidegger, G. (2005): Evaluationsforschung. In: Rauner, F. (Hrsg.): Handbuch Berufsbildungsforschung. Bielefeld: Bertelsmann, S. 412-421.

Heinen, S. (2012): Studien der Qualifikationsforschung in der Domäne der Gebäudeenergieberatung. In: Reichwald, R./Frenz, M./Hermann, S./Schipanski, A. (Hrsg.): Zukunftsfeld Dienstleistungsarbeit. Wiesbaden: Gabler, S. 281-302.

Heinen, S./Frenz, M./Djaloeis, R./Schlick, C. (2011): Analytische und konzeptionelle Überlegungen für Fort- und Weiterbildungen in der Gebäudeenergieberatung. In: Frenz, M./Unger, T./Schlick, C. (Hrsg.): Moderne Beruflichkeit. Bielefeld: Bertelsmann, S.23-48.

Heinen, S./Frenz, M./Schlick, C. (2013): Bildung für nachhaltige Entwicklung in der Gebäudeenergieberatung - Entwicklung eines Kompetenzmodells und Konzeption von Weiterbildungsmodulen. In: Becker, M./Grimm, A./Petersen, W./ Schlausch, R. (Hrsg.): Kompetenzorientierung und Strukturen gewerblich-technischer Ausbildung. Berlin: Lit, S. 456-473.

Kandler, M./Tippelt, R. (2011): Weiterbildung und Umwelt: Bildung für nachhaltige Entwicklung. In: Tippelt, R./Hippel, A. (Hrsg.): Handbuch Erwachsenenbildung/ Weiterbildung. Wiesbaden: Springer, S. 707-728.

Reetz, L. (1999): Zum Zusammenhang von Schlüsselqualifikationen - Kompetenzen - Bildung. In: Tramm, T. (Hrsg.): Professionalisierung kaufmännischer Berufsbildung. Frankfurt: Peter Lang.

Reetz, L. (2011): Untersuchungen zur Praxis der Erfassung beruflicher Handlungskompetenz bei den Abschlussprüfungen im dualen System der deutschen Berufs- 
ausbildung. In: Münk, D./Schelten, A. (Hrsg.): Kompetenzermittlung für die Berufsbildung. Bielefeld: Bertelsmann, S. 101-118.

Reetz, L./Seyd, W. (2006): Curriculare Strukturen beruflicher Bildung. In: Arnold, R./Lipsmeyer, A. (Hrsg.): Handbuch der Berufsbildung. 2. Aufl. Wiesbaden: Springer, S. 227-259.

Roth, H. (1976): Pädagogische Anthropologie, Band II, 2. Aufl. Hannover: Schroedel. Siebert, H. (2012): Didaktisches Handeln in der Erwachsenenbildung. 7. Aufl. Augsburg: Ziel.

Tiefel, S. (2004): Beratung und Reflexion - eine qualitative Studie zu professionellem Beratungshandeln in der Moderne. Wiesbaden:VS. 